\title{
Effect of Image Resolution on Remote Photoplethysmography: Towards Emotion Detection in Children with Autism Spectrum Disorder
}

\author{
Lucas Lampier* Alan Floriano* Denis Delisle-Rodriguez* \\ Eliete Caldeira ${ }^{* *}$ Teodiano Bastos-Filho* \\ * Postgraduate Program in Electrical Engineering, Federal University of \\ Espirito Santo,UFES, (e-mails: lucas.lampier@hotmail.com; \\ afloriano.ufes@gmail.com; delisle05@gmail.com; \\ teodiano.bastos@ufes.br). \\ ** Department of Electrical Engineering, Federal University of Espirito \\ Santo,UFES, (e-mail: eliete.caldeira@ufes.br).
}

\begin{abstract}
Aiming to stimulate social abilities in children with Autism Spectrum Disorder was developed at UFES/Brazil a robot to interact with them. One of the functions that are being developed in that robot is emotion recognition using cardiac activity. Traditional heart rate measuring methods normally use contact sensors, often uncomfortable for autistic children. So, a method to measure it remotely and online is an important contribution to this field of research. This paper presents a method to remotely measure heart rate using a low-cost RGB camera. The effect of the different image resolutions in the final result is evaluated, aiming in a future online system, which needs a small computation time. The results indicate that the resolution does not have a relevant influence on measure's precision, so, the resolution of the video can be reduced without a big effect on the error.
\end{abstract}

Keywords: Autism; Child-Robot Interaction; Remote Photoplethysmography; Remote Heart Rate; RGB camera; Resolution.

\section{INTRODUÇÃO}

According to Klin (2006), autism spectrum disorder (ASD) is a clinical condition in a group known as Pervasive Developmental Disorder (PDD). The PDDs are neurological disorders in nature, characterized by the early onset of delays and deviations from various abilities, mainly social and communicative. Commonly, the typical characteristic of the autistic spectrum appear before three years of life.

Statistics from the United Nations reported in Stock (2018) show that are about 70 million autistic children in the World (data for 2018), in majority boys. Brazil does not have official statistics, but it is reported that the number is close to 2 million.

Aiming to stimulate attention, some social skills and the capacity of interaction with the environment of autistic children, the robot MARIA (Mobile Autonomous Robot for Interaction with Autistics) was developed at UFES/Brazil. The robot built with a PIONEER 3-DX, has a funny design to attract children's attention, a multimedia system for audiovisual interaction, a system of RGB and thermographic cameras to capture images of the face, and a

\footnotetext{
* The authors thank CNPq - Conselho Nacional de Desenvolvimento Científico e Tecnológico, FAPES - Fundação de Amparo à Pesquisa e Inovação do Espírito Santo and CAPES - Coordenação de Aperfeiçoamento de Pessoal de Nível Superior - for the financial support granted to this work
}

laser sensor to get the child's location and keep a safe distance. A new version of the robot, called N-MARIA (New-MARIA), with a better design, is shown in Figure 1. For more information about the robot see Valadão et al. (2016).

The research conducted by Goulart et al. (2015) shows that the interaction between ASD children and this kind of robot may trigger increase of interest in the child, in addition to a wide variety of social skills, such as eye contact and imitation. The results of the research carried show that the interaction between the robot MARIA and the ASD children was better than expectation. They touched, looked more closely at the robot and interacted with the mediator more than expected. Only one ASD child showed fear of interacting with the robot.

One of the objectives of this work is to measure the interaction level between child and robot by capturing the emotional state of the child. One of the parameters that can be used for that is the Heart Rate Variability (HRV), which is highly linked to emotional intensity according to Appelhans and Luecken (2006).

However, one problem of measuring cardiovascular parameters is that traditional methods to measure the Heart Rate (HR) generally use sensors in contact with the skin, like electrodes. According to Allen (2007), alternative ways, such the Photoplethysmography (PPG), can mea- 


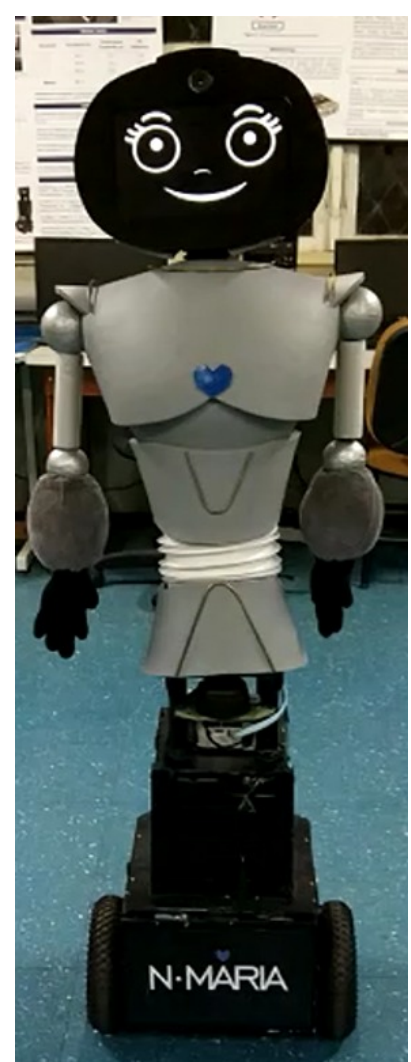

Figure 1. Robot developed at UFES/Brazil N-MARIA, the newer version of the robot MARIA.

sure it using optical devices that detect variation in blood flow.

In the last decade the development in hardware in addition to techniques for image and signal processing allowed measuring HR using an ordinary RGB camera by capturing the color changes caused by the variability of the blood volume under the skin. Research conducted by Caseiro et al. (2012), McDuff et al. (2018) and Li et al. (2014) show different ways to measure HR using a low cost RGB camera. The review work done by Rouast et al. (2018) reports that most authors call this technique as remote photoplethysmography (rPPG).

Thus, rPPG could be incorporated into the N-MARIA robot to measure children's cardiac parameters as they interact with the robot, without any contact sensors.

The work done by Rouast et al. (2018) presents an overview in the state of the art in the use of low cost RGB cameras in rPPG. Different techniques of extraction, tracking and stabilization of regions of interest (ROIs) are shown, as well as, the regions of the face where the variation and blood flow is best captured, the color channels used to generate the signal, the main filtering techniques and signal processing used for $\mathrm{HR}$ calculations. In the review only three articles, have presented a method for online measuring. See Tran et al. (2015), Li and Lin (2015), Rouast et al. (2017).

One of the limitations for the system to work online is the signal extraction and processing time. Considering that one of the most time consuming parts of the processing is the face tracking and image processing of the frames, the resolution of the image tends to have a considerable impact on the execution time. Thus, decreasing the image resolution decreases the number of pixels to be processed and, consequently, the time spent in calculations. On the other hand, the decrease of the resolution impacts on the quality of the image, which can interfere directly in the error during the calculations. Thus, this work compares the error obtained during the calculation of rPPG using different video resolutions.

This paper is subdivided in four sections. First, in "Introduction" a general idea of the motivation and objectives of this work is given. In "Materials and Methods", the steps used to acquire the data, process the signals and calculate the HR are presented. In "Results and Discussion", the error obtained using different video resolutions for each subject are shown. And, finally, in "Conclusions", the analysis done over the reached results is presented.

\section{MATERIALS AND METHODS}

The main process to infer the HR using a webcam is shown in Figure 2. The first step is to select regions of the frame that contain the subject's skin. To accomplish that, the location of the face in the frame is needed, so the following algorithms are used to detect and track the face along the video: the Viola-Jones (VJ) and the Kernelized Correlation Filter (KCF). To select the skin from the face, a sample of the skin's tone of the user is extracted when the face is detected, which is used to define the color limits to extract the skin in the following frames.

After identifying the pixels containing skin, the average value of the RGB intensity of these pixels is calculated, then they are used to calculate the POS (PlaneOrthogonal-to-Skin) signal. A Butterworth filter is used to attenuate the frequency components that are off the limits of the common HR values. And finally, the Lomb-Scargle Periodogram (LSP) is used to find the frequency with the highest magnitude as the HR value. The VJ was proposed by Viola and Jones (2001), the KCF by Henriques et al. (2015), both of them are available in Bradski (2000). The The overall steps of the proposed methodology can be seen in Figure 2.

To compare the results, the execution time, the error, and the average of Signal-to-Noise Ratio (SNR) obtained using each video resolution were recorded.

\subsection{Data acquisition protocol}

The experiments were performed with three volunteers, aged between 24 and 27 years, all males. Volunteers 1 and 2 have a beard, while Volunteer 3 does not. As for skin color, Volunteers 1 and 2 have white skin, and Volunteer 3 has slightly darker skin. This research was approved by the ethics committee of UFES (CAAE $\mathrm{n}^{\circ}$ 44899015.0.0000.5060).

During the experiments, each volunteer should remain static, seated in front of the camera for 5 min at a distance of approximately $1 \mathrm{~m}$ from the camera. To validate the results, the ECG signal was captured using the BrainNet equipment (EMSA). Both RGB video and ECG were simultaneously recorded, as ECG is a gold standard for HR studies. 


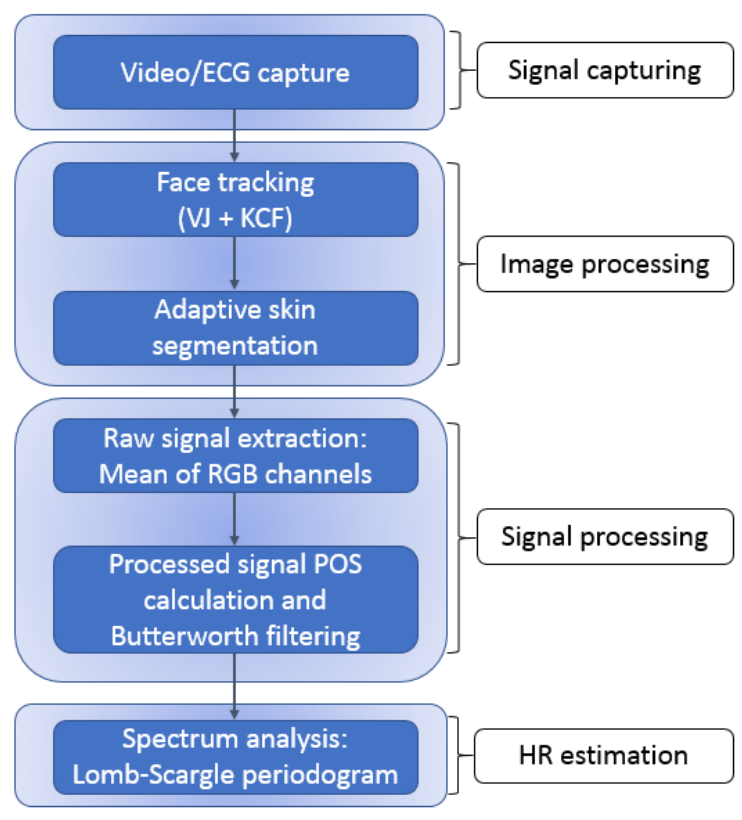

Figure 2. Fluxogram with the methodology steps.

Each video is recorded in 640 pixels of width and 480 pixels of height. For the experiments three resolution sizes are used, $640 \times 480$ (the original), $320 \times 240$ and $160 \times 120$. The interpolation method used is the bilinear interpolation, from the OpenCV library.

During the experiments the videos are recorded at an average acquisition rate of $15 \mathrm{fps}$, as the ambient light intensity is approximately 190 lux. The ECG signal is captured in a frequency range from 0.15 to $150 \mathrm{~Hz}$, and sampling rate at $400 \mathrm{~Hz}$. The D1 derivation is measured, according to Pastore et al. (2009).

\subsection{Face Track}

The face tracking method applied has three steps: initialization, tracking and recalibration. The initialization step consists of using the VJ to identify the position and size of the subject's face in the first frame. The tracking step consists of inserting the parameters of the rectangle containing the face found in the tracking algorithm (KCF), to follow the face along the video. However, the algorithm may lose the location of the face, especially in cases of occlusion. To avoid it, the recalibration part is called to reset the tracker algorithm every 150 frames (approximately $10 \mathrm{~s}$ ). Its function is to execute the $\mathrm{VJ}$ to locate the user's face again in the frame and, if it is found, the tracker is reconfigured with the new parameters. If the face is not found in the current frame, due to occlusion or face rotation for example, the tracker keeps its information until the VJ can find the face in another frame, and then the $\mathrm{KCF}$ is reinitialized with the new VJ results.

\subsection{Skin Segmentation}

The skin segmentation technique is based on the method presented by Badwaik and Lokhande (2018), but instead of using the $\mathrm{YCgCr}$ color space, the $\mathrm{YCrCb}$ color system is chosen, as the transition between $\mathrm{RGB}$ and $\mathrm{YCrCb}$ is already provided in OpenCV and, according to Bousefsaf et al. (2013) $Y C r C b$ system has shown success in detecting skin. The method proposed by Badwaik and Lokhande (2018) selects a sample of the image that contains the subject skin, then take its values to define the limits to select the skin pixels to the whole frame. So, we took advantage that the VJ code is used to detect the face, and use the tool provided by Sagonas et al. (2015), executed with the Dlib library provided by King (2009), to detect the facial landmarks (specific points in the face, which is presented with the red points in Figure 3) and then take the skin samples of the left and right cheeks and forehead (Figure 3).

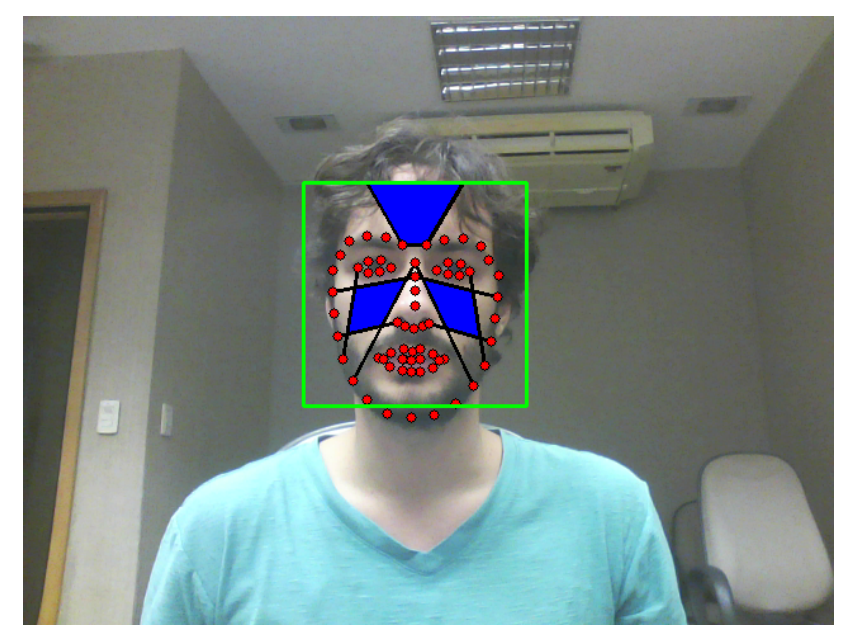

Figure 3. In blue are the skin regions taken as sample to define the color limits of the skin segmentation method. The dots in red are the facial landmarks returned by the trained code from Sagonas et al. (2015). The green rectangle is the face region returned by the VJ algorithm.

Then, the $Y C r C b$ values of each sample are sorted, and the $7.5 \%$ ones with larger and smaller intensities of each channel are removed. Such removal lessens the chance of non-skin components affect the result, such as glasses or facial hair. Then the lowest value of each channel of the three regions are taken as the minimum limit, and the highest value as the maximum limit. Finally, all pixels inside the rectangle of the face that are within the limits are selected as skin pixels. To remove the pixels corresponding to the region of the mouth, decreasing the influence of the mouth movement, the pixels of the lower part (20\% of the height of the rectangle) were eliminated. Figure 4 presents the skin region selected by the algorithm.

Finally, the average value of the RGB channels from the skin pixels are calculated and stored in a $C_{N \times 3}$ matrix to compose a temporal signal, where $N$ is the number of frames processed, and the columns represent each color channel.

\section{4 rPPG Signal Calculation and HR Estimation}

After calculating the $C$ matrix, the first step signal processing is to normalize the signal and remove the offset component using equation (1), according to Wang et al. (2017). 


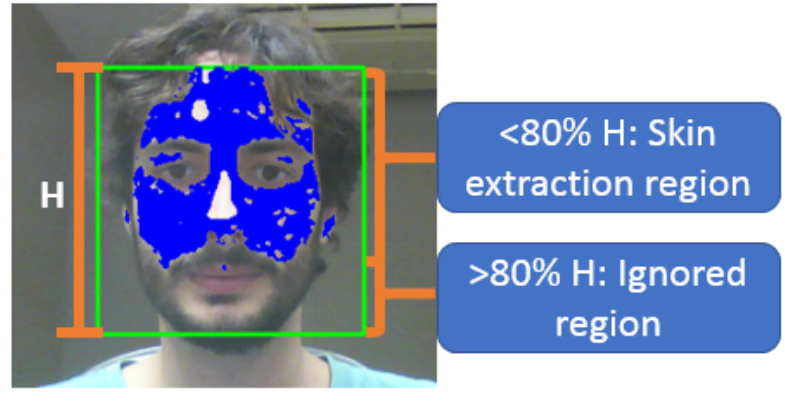

Figure 4. Result of the skin segmentation method. In blue are the skin's pixels selected, and the green rectangle represents the face region returned by the tracker.

$$
\tilde{C}_{i}=\frac{C_{i}}{\mu\left(C_{i}\right)}-1,
$$

Where $i$ represents the $i^{\text {th }}$ color channel and $\mu($.$) repre-$ sents the average operation.

After normalizing the signal, equation (2) is used to calculate the POS, proposed by Wang et al. (2017) to extract the blood variation signal.

$$
P=X+\alpha Y ; \quad \text { with }\left\{\begin{array}{l}
X=G-B, \\
Y=G+B-2 R \\
\alpha=\frac{\sigma(X)}{\sigma(Y)}
\end{array}\right.
$$

Then the frequency components of the POS signal outside the range of interest (the frequencies that do not represent the common HR of a person in a normal state) are removed using a fifth order passband Butterworth filter with cutoffs frequencies at $0.6 \mathrm{~Hz}$ and $3 \mathrm{~Hz}$ (equivalent to 36 to 180 bpms).

To find the corresponding HR of the filtered POS signal, a spectrum analysis tool is used. Instead using the Fast Fourier Transform (FFT) that needs a constant frequency sampled signal, the analysis technique used in this work is the same as that used in Chen et al. (2018), the Lomb-Scargle periodogram, which is a frequency analysis technique that considers a variable sampling rate. Thus the frequency of higher power within the spectrum of interest is considered as the heart rate for the window of analysis. Calculations are done using a window of $30 \mathrm{~s}$, with overlap of $29 \mathrm{~s}$. Figure 5 shows the results of the signal processing step.

\subsection{Calculating HR from the ECG}

The Python BioSppy library developed by Carreiras (2015) is used to locate the position of the $\mathrm{R}$ peaks of the QRS complex of the ECG signal. To calculate the HR, the time differences between consecutive peaks in a $30 \mathrm{~s}$ window are taken. Then the inverse of these differences peaks are calculated to obtain the HR value (in Hertz). The result is multiplied by 60 to obtain the measure in beats per minute (bpm). Finally, the average of the value in bpm is calculated.
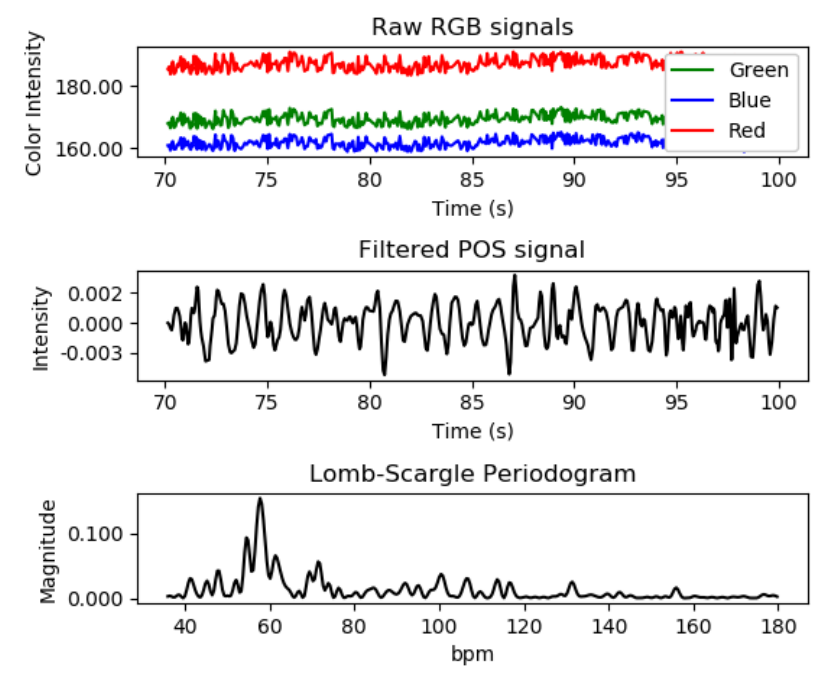

Figure 5. Representation of the signal processing step. Starting with the raw RGB signal captured from the skin pixels, then the respective processed POS signal, and finally, the power spectrum of the signal with the peak, approximately on $60 \mathrm{bpm}$, indicating the HR measured.

\section{RESULTS AND DISCUSSION}

To compare the elapsed time obtained using the three proposed image sizes, for each resolution, the running time to process the whole video was recorded. In order to compare the results with a gold standard method, the difference between the HR values calculated by video and by ECG was measured using two metrics: the Root Square Mean Error (RMSE) and mean error with the standard deviation. To analyze the noise generated by each resolution, the mean SNR for each window is calculated, in same way as Wang et al. (2017), using the power of the frequencies inside the range of the HR found by the ECG $\pm 6 \mathrm{bpms}$, divided by the power of whole spectrum. The result of the error calculations and the mean SNR can be seen in Table 1. The spectrogram of the signals are shown in Figure 6.

Table 1. Errors and SNR for each subject and resolution combination, including the overall results with the mean values for each resolution.

\begin{tabular}{lccccc}
\hline \multicolumn{1}{c}{ Subject } & $\begin{array}{c}\text { WxH } \\
\text { (Pixels) }\end{array}$ & $\begin{array}{c}\text { Time } \\
(\mathrm{s})\end{array}$ & $\begin{array}{c}\text { RMSE } \\
(\mathrm{bpm})\end{array}$ & $\begin{array}{c}\text { Mean } \pm \text { STD } \\
(\mathrm{bpm})\end{array}$ & $\begin{array}{c}\text { Mean } \\
\text { SNR } \\
(\mathrm{dB})\end{array}$ \\
\hline S. 0 & $640 \times 480$ & 282.47 & 5.16 & $2.05 \pm 4.79$ & -5.81 \\
S. 0 & $320 \times 240$ & 292.30 & 4.03 & $1.56 \pm 3.76$ & -5.61 \\
S. 0 & $160 \times 120$ & 251.90 & 2.72 & $1.37 \pm 2.38$ & -5.53 \\
\hline S. 1 & $640 \times 480$ & 323.44 & 2.10 & $-0.03 \pm 2.10$ & -4.15 \\
S. 1 & $320 \times 240$ & 274.87 & 2.34 & $0.12 \pm 2.34$ & -4.11 \\
S. 1 & $160 \times 120$ & 223.27 & 3.87 & $0.86 \pm 3.78$ & -4.39 \\
\hline S. 2 & $640 \times 480$ & 266.98 & 8.13 & $6.01 \pm 5.59$ & -6.66 \\
S. 2 & $320 \times 240$ & 253.92 & 7.47 & $5.00 \pm 5.66$ & -6.23 \\
S. 2 & $160 \times 120$ & 301.01 & 7.83 & $5.83 \pm 5.35$ & -5.99 \\
\hline Overall & $640 \times 480$ & 290.96 & 5.13 & $2.68 \pm 4.16$ & -5.54 \\
Overall & $320 \times 240$ & 273.70 & 4.62 & $2.22 \pm 3.92$ & -5.32 \\
Overall & $160 \times 120$ & 258.73 & 4.81 & $2.69 \pm 3.84$ & -5.30 \\
\hline
\end{tabular}



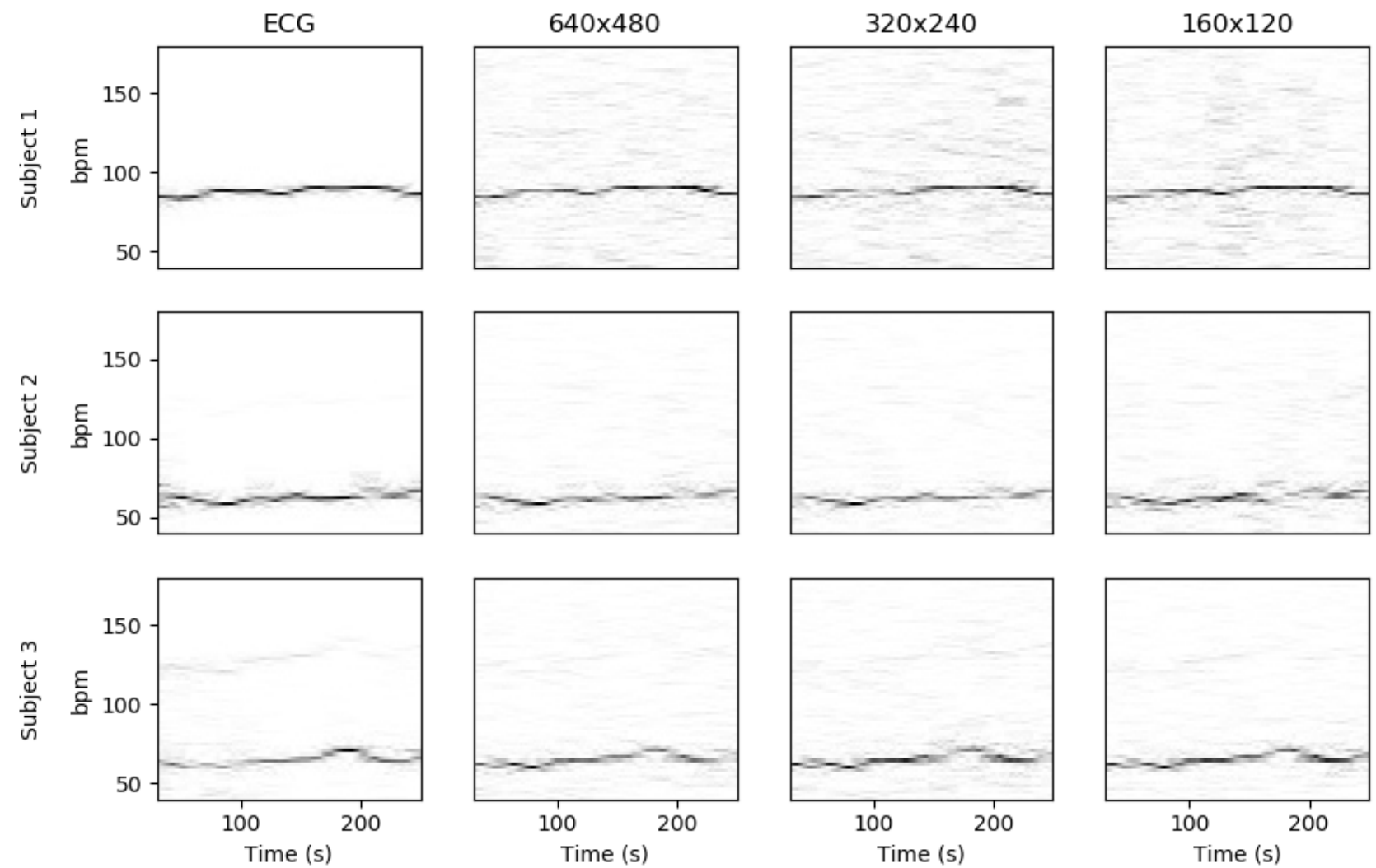

Figure 6. Spectogram showing the power spectrum of the ECG signal and the rPPG signal for each subject in each resolution.

The error between the three resolutions in Table 1 are really close to each other. Looking to the overall RMSE, the difference between the worst value (resolution 160x120) and the best one $(640 \times 480)$ is 0.32 bpms, and the SNR difference between the worst $(640 \times 480)$ and the best one $(160 \times 120)$ is $0.24 \mathrm{~dB}$. That indicates that the size reduction does not have a negative impact on the result. The spectrogram of the ECG and POS signal calculated from the video signals is shown in Figure 6. It is possible to see that, the bpm line (the darker line) has the same format in all signals, but the spectogram from the video signals are noisier. In the other hand, by decreasing the resolutions, the processing time was reduced by more than 30 s from the $640 \times 480$ resolution to the $160 \times 120$ comparing the overall values.

\section{CONCLUSIONS}

Focusing on developing an on-line rPPG for a child-robot interaction, which has limited hardware to process data, this work compared the error resulted from different image resolution in the estimation of the HR using an notebook integrated webcam. The results showed that as long as the skin can be rightly selected from the image, the resolution has a small impact on the error obtained and the SNR of the signal. So, a smaller image can be used in the calculus, reducing the processing time of the software and keeping the quality of the measurements. The next step of this work is to conduct experiment with ASD children.

\section{REFERENCES}

Allen, J. (2007). Photoplethysmography and its application in clinical physiological measurement. Physiological Measurement, 28(3), R1-R39. doi:10.1088/0967-3334/ $28 / 3 / \mathrm{r} 01$.

Appelhans, B.M. and Luecken, L.J. (2006). Heart rate variability as an index of regulated emotional responding. Review of General Psychology, 10(3), 229-240. doi: 10.1037/1089-2680.10.3.229.

Badwaik, S.C. and Lokhande, S.D. (2018). Adaptive skin colour modelling for hand and face segmentation. International Journal of Intelligent Engineering and Systems, 11(5), 84-95. doi:10.22266/IJIES2018.1031.08.

Bousefsaf, F., Maaoui, C., and Pruski, A. (2013). Continuous wavelet filtering on webcam photoplethysmographic signals to remotely assess the instantaneous heart rate. Biomedical Signal Processing and Control, 8(6), 568574. doi:10.1016/j.bspc.2013.05.010.

Bradski, G. (2000). The OpenCV Library. Dr. Dobb's Journal of Software Tools.

Carreiras, C. (2015). BioSPPy: Biosignal processing in Python. URL https://github.com/PIA-Group/ BioSPPy.

Caseiro, R., Martins, P., and Batista, J. (2012). Exploiting the Circulant Structure of Tracking-by-detection with Kernels. Eccv, 50, 4-6. doi:10.1007/978-3-642-33765-9_ 50.

Chen, W., Hernandez, J., and Picard, R.W. (2018). Estimating carotid pulse and breathing rate from nearinfrared video of the neck. Physiological Measurement, 
39(10), 10NT01. doi:10.1088/1361-6579/aae625.

Goulart, C., Valadão, C., Caldeira, E.M.O., and BastosFilho, T.F. (2015). MARIA: Um Robô para Interação com Crianças com Autismo. XII Simpósio Brasileiro de Automação Inteligente (SBAI), 557-562.

Henriques, J.F., Caseiro, R., Martins, P., and Batista, J. (2015). High-speed tracking with kernelized correlation filters. IEEE Transactions on Pattern Analysis and Machine Intelligence, 37(3), 583-596. doi:10.1109/TPAMI. 2014.2345390.

King, D.E. (2009). Dlib-ml: A machine learning toolkit. Journal of Machine Learning Research, 10, 1755-1758.

Klin, A. (2006). Autismo e síndrome de Asperger: uma visão geral Autism and Asperger syndrome: an overview. Rev Bras Psiquiatr, 28(Supl I), 3-11.

Li, M. and Lin, Y. (2015). A real-time non-contact pulse rate detector based on smartphone. In 2015 International Symposium on Next-Generation Electronics (ISNE), 1-3. doi:10.1109/ISNE.2015.7132025.

Li, X., Chen, J., Zhao, G., and Pietikäinen, M. (2014). Remote heart rate measurement from face videos under realistic situations. Proceedings of the IEEE Computer Society Conference on Computer Vision and Pattern Recognition, 4264-4271. doi:10.1109/CVPR.2014.543.

McDuff, D.J., Blackford, E.B., and Estepp, J.R. (2018). Fusing partial camera signals for noncontact pulse rate variability measurement. IEEE Transactions on Biomedical Engineering, 65(8), 1725-1739. doi:10.1109/ TBME.2017.2771518.

Pastore, C., Pinho, C., Germiniani, H., Samesima, N., and Mano, R. (2009). Diretrizes da sociedade brasileira de cardiologia sobre Ánalise e emissão de laudos eletrocardiográficos. Arquivos Brasileiros de Cardiologia, 93, 1 19.

Rouast, P.V., Adam, M.T.P., Chiong, R., Cornforth, D., and Lux, E. (2018). Remote Heart Rate Measurement Using Low-Cost RGB Face Video: A Technical Literature Review. Frontiers of Computer Science, 12(5), 858-872. doi:10.1007/s11704-016-6243-6.

Rouast, P.V., Adam, M.T.P., Cornforth, D.J., Lux, E., and Weinhardt, C. (2017). Using contactless heart rate measurements for real-time assessment of affective states. In F.D. Davis, R. Riedl, J. vom Brocke, P.M. Léger, and A.B. Randolph (eds.), Information Systems and Neuroscience, 157-163. Springer International Publishing, Cham.

Sagonas, C., Antonakos, E., Tzimiropoulos, G., Zafeiriou, S., and Pantic, M. (2015). 300 Faces In-The-Wild Challenge: database and results. Image and Vision Computing, 47, 3-18. doi:10.1016/j.imavis.2016.01.002.

Stock, A. (2018). Quais são as teorias e as pesquisas sobre as possíveis causas do autismo. URL http://www.bbc . com/portuguese/geral-43577510.

Tran, D.N., Lee, H., and Kim, C. (2015). A Robust Real Time System for Remote Heart Rate Measurement via Camera. Proceedings - IEEE International Conference on Multimedia and Expo, 2015-Augus, 1-6. doi:10.1109/ ICME.2015.7177484.

Valadão, C.T., Goulart, C., Rivera, H., Caldeira, E., Bastos Filho, T.F., Frizera-Neto, A., and Carelli, R. (2016). Analysis of the use of a robot to improve social skills in children with autism spectrum disorder. Research on Biomedical Engineering, 32, 161 - 175. doi:10.1590/
2446-4740.01316.

Viola, P. and Jones, M. (2001). Rapid object detection using a boosted cascade of simple features. Proceedings of the 2001 IEEE Computer Society Conference on Computer Vision and Pattern Recognition. CVPR 2001, 1, I-511-I-518. doi:10.1109/CVPR.2001.990517.

Wang, W., Den Brinker, A.C., Stuijk, S., and De Haan, G. (2017). Algorithmic principles of remote ppg. IEEE Transactions on Biomedical Engineering, 64(7), 14791491. doi:10.1109/TBME.2016.2609282.

Wang, W., Den Brinker, A.C., Stuijk, S., and De Haan, G. (2017). Robust heart rate from fitness videos. Physiological Measurement, 38(6), 1023-1044. doi:10. 1088/1361-6579/aa6d02. 